\title{
What makes a density functional approximation good? Insights from the left Fukui function
}

\author{
Tim Gould* \\ Qld Micro- and Nanotechnology Centre, Griffith University, Nathan, Qld 4111, Australia \\ E-mail: t.gould@griffith.edu.au
}

\begin{abstract}
This manuscript proposes, justifies and reports tests of the chemically-relevent left Fukui function of Li, C and F on a range of density functional approximations. Analysis indicates that functionals can be good at densities and bad at Fukui functions, and vice versa, analogous with energies and ionisation potentials. "TPSSh", "SOGGA11X" and "B2PLYP" are star performers on both, however. Many "Minnesota functionals" fare much better here than in a recent analysis of electron densities. In this context, new optimizing strategies are mentioned.
\end{abstract}

Density functional theory ${ }^{1,2}$ (DFT) has shown outstanding success in chemistry and physics over the past three decades. Modern codes and density functional approximations (DFAs) have made DFT modelling an important supplement to experiments and fundamental theory across many areas of chemistry and physics. It is fair to say that DFT has transformed quantitative understanding of chemical systems by making calculation of their energetics both possible and reliable.

However, this success has led DFT to evolve into a myriad of forms. The number of available "functionals" (DFAs) has grown alongside the number of applications of DFT. DFAs

*To whom correspondence should be addressed 
could be said to have evolved into two (overlapping) families: constrained approaches which obey increasing numbers of exact conditions, and (semi-)empirical approaches which are fit to large numbers of test cases. The number of inputs has also grown, ${ }^{3}$ from approximations based on the density $n$ (LDAs) only, to including its gradient (GGAs), the exchange energy (hybrids, range-separated hybrids), and the kinetic energy density (meta-GGAs).

Consequently, users can now choose from a long list of approximations, or even a continuum when approaches with free parameters are thrown into the mix. For meaningful results users must thus choose an appropriate approximation when carrying out their modelling. But how should an approximation be chosen? Which approximation is best?

The above (and titular) questions, of course, lack clear answers. The relative "good" ness of different approximations can be determined according to numerous metrics. Most commonly they are rated on how well they perform for energy differences within chemical or material science problems (e.g. for reaction energies or atomization energies); sometimes balanced against their numerical cost (LDAs and GGAs are usually faster than meta-GGAs and much faster than hybrids). But DFAs are sometimes chosen for other reasons, like accurate prediction of band-structures. ${ }^{4,5}$

A recent manuscript ${ }^{6}$ adopted a novel approach to the question. The authors tested 128 density functional approximations on their ability to closely reproduce the density of 14 atoms and cations with 2, 4 and 10 electrons. Accurate density benchmarks were obtained using CCSD theory and compared to densities obtained self-consistently using approximations. Their core conclusion was rather shocking: modern "empirical" approximations that are optimized on energies in real systems can yield densities that are signficantly worse than approaches that are based on semi-homogenous electron gases. Given that DFT uses the density as the basic variable, this might be viewed as an alarming finding. [Although we note recent comments by Kepp ${ }^{7}$ and response by Medvedev et al. $\left.{ }^{8}\right]$

As shall be argued below, however, that study had two fundamental methodological biases that may have skewed it towards a certain class of density functional approximations. 
Its applicability to actual chemical problems is thus questionable, as is the relevance of its conclusions.

To enable a fairer analysis, this manuscript first identifies, and seeks to address these methodological biases to provide a chemically relevant metric for different approximations (DFAs). Then, results are reported for a number of modern functionals tested using the new metric. Finally, conclusions are drawn.

Bias 1: Medvedev et $a l^{6}$ tested 14 closed shell atoms and (up to 8+) cations with 2, 4 and 10 electrons. But closed shell atoms have relatively simple quantum physics compared to open shell atoms and molecules. On the one hand, their electrons are bound tightly to the nucleus even in the neutral atoms (except Be), and especially in the cations, as evidenced by their high ionization potentials. This did not present a problem for Medvedev et $a l^{6}$ whose work/philosophy focused on the ability of DFAs to reproduce atomic density "norms", as elaborated in their later response to Kepp. ${ }^{8}$ However, the selection does bias results to the high-density limit where certain functionals (e.g., PBE, ${ }^{9}$ or $\operatorname{TPSS}^{10}$ ) which include many limiting constraints on properties are designed to behave well. ${ }^{11}$ On the other hand, the atoms are in high symmetry states. "Empirical" approximations that are optimized to reproduce global energy properties (e.g., the Minnesota functionals), including binding energies, may not fully showcase their virtues in closed-shell atoms and cations. Analysis was therefore skewed to favour "non-empirical" approaches constrained and/or optimized to the appropriate limit(s).

Solution 1: Rather than a largely chemically-irrelevant set of closed-shell atoms and cations, with consequent biases, this work focuses on open-shell atoms Li, C and F. These three atoms provide good benchmarks for chemical reactions. Li and F serve as exemplar electron donors and receptors respectively. $\mathrm{C}$ is the most abundant element in chemistry and is thus the element most likely to dominate in optimizations over molecular properties. Furthermore, high quality reference density data (parametrised from quantum-monte Carlo data using exact constraints) is available ${ }^{12}$ for all three atoms and their singly positive cations. 
Thus chemically-relevant density differences (the Fukui function, see below) can be tested for the three elements to study the important electron binding physics captured by DFAs.

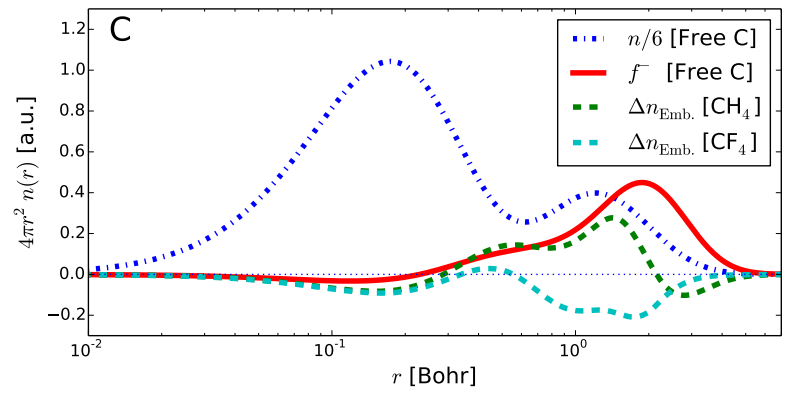

Figure 1: The Fukui function $f^{-}$is comparable to the difference $\Delta n_{\text {Emb. between a free atom }}$ density and an embedded (in $\mathrm{CH}_{4} / \mathrm{CF}_{4}$ ) atom density, highlighting its chemical importance. This is in contrast to the total density.

Bias 2: Previous analysis tested absolute densities, including bonding and core regions. But it is well known that DFAs typically give better energy differences than absolute energies. Indeed modern functionals are often optimized ${ }^{13-15}$ for this purpose. When it comes to densities, one might expect a similar relationship to hold. In particular, optimized approaches might be expected to favour accurate reproduction of binding regions, at the sacrifice of accurate densities for the largely unimportant nuclear region of constituent parts of molecules. This crucial distinction cannot be captured when only absolute densities are analysed, as done by Medvedev et al. Especially not when core contributions are further amplified by the inclusion of gradients and Laplacians of the density in tests (see Section IA of the Supporting Information for further details).

Solution 2: This work focuses on the ability of DFAs to reproduce density differences: specifically, their ability to reproduce the chemically-relevant Fukui function $f^{-}(\mathbf{r})=n^{\text {atom }}(\mathbf{r})-$ $n^{\text {cation }}+(\mathbf{r})$ [see Eqn. 3], which is typically similar to ${ }^{16}$ (but not the same as, see also Supporting Information) the density of the frontier orbital from DFT obtained from Kohn-Sham ${ }^{2}$ or generalized Kohn-Sham ${ }^{17}$ theory. It also excludes gradients and Laplacians to avoid bias to core regions. Not only is the Fukui function more in keeping with the spirit of most applications of DFT; but it tests the ability of approximations to reproduce properties of 
similar systems with locally different numbers of electrons and is thus more broadly relevant to chemistry.

The importance of using the Fukui function ${ }^{18}$ is highlighted in Figure 1, where the density change $\Delta n_{\text {Emb. }}(r)$ of a $\mathrm{C}$ atom embedded in $\mathrm{CH}_{4}$ and $\mathrm{CF}_{4}$ is compared to the exact radially averaged Fukui function

$$
f^{-}(r)=\int \frac{d \hat{\mathbf{r}}}{4 \pi r^{2}}\left[n^{\text {atom }}(\mathbf{r})-n^{\text {cation }^{+}}(\mathbf{r})\right]
$$

Here vector $\mathbf{r}$ is defined relative to the $\mathrm{C}$ nucleus. $\Delta n_{\mathrm{Emb}}(r)$ is the radial average of the Hirshfeld partitioned ${ }^{19} \mathrm{C}$ atom in $\mathrm{CH}_{4} / \mathrm{CF}_{4}$ (using B3LYP ${ }^{20}$ ) minus its free atom equivalent. It is clear that the Fukui function, unlike the density, is dominant in precisely the regions where the density changes from embedding are dominant - and is thus a good measure of chemical changes to the density. The Fukui function is able to capture some of the physics involved in bonding using only atomic/ionic properties. It thus avoids the "understood but uncontrollable error cancellation" ${ }^{6}$ that makes analysis of molecules difficult.

Analysis: Now that biases have been identified in previous analytic techniques, and techniques have been proposed that avoid them, it is possible to investigate the quality of a number different DFAs. The test set includes 37 popular functionals available in Gaussian 16. It focuses on approaches of the "Minnesota family" ${ }^{21}$ as these are typically more "empirical" (in the sense that they are heavily parametrised) and were identified as problematic in previous work. ${ }^{6}$ A full list of DFAs is included in the supplementary material, along with Gaussian keywords and references. Some DFAs were difficult (M11L, SOGGA11, MN12SX) or impossible (MN12L) to converge for atomic Li. Caution is thus recommended for using these functionals outside their suggested domain (see also Supporting Information).

The analysis uses the following metrics for errors in the density and Fukui function, 

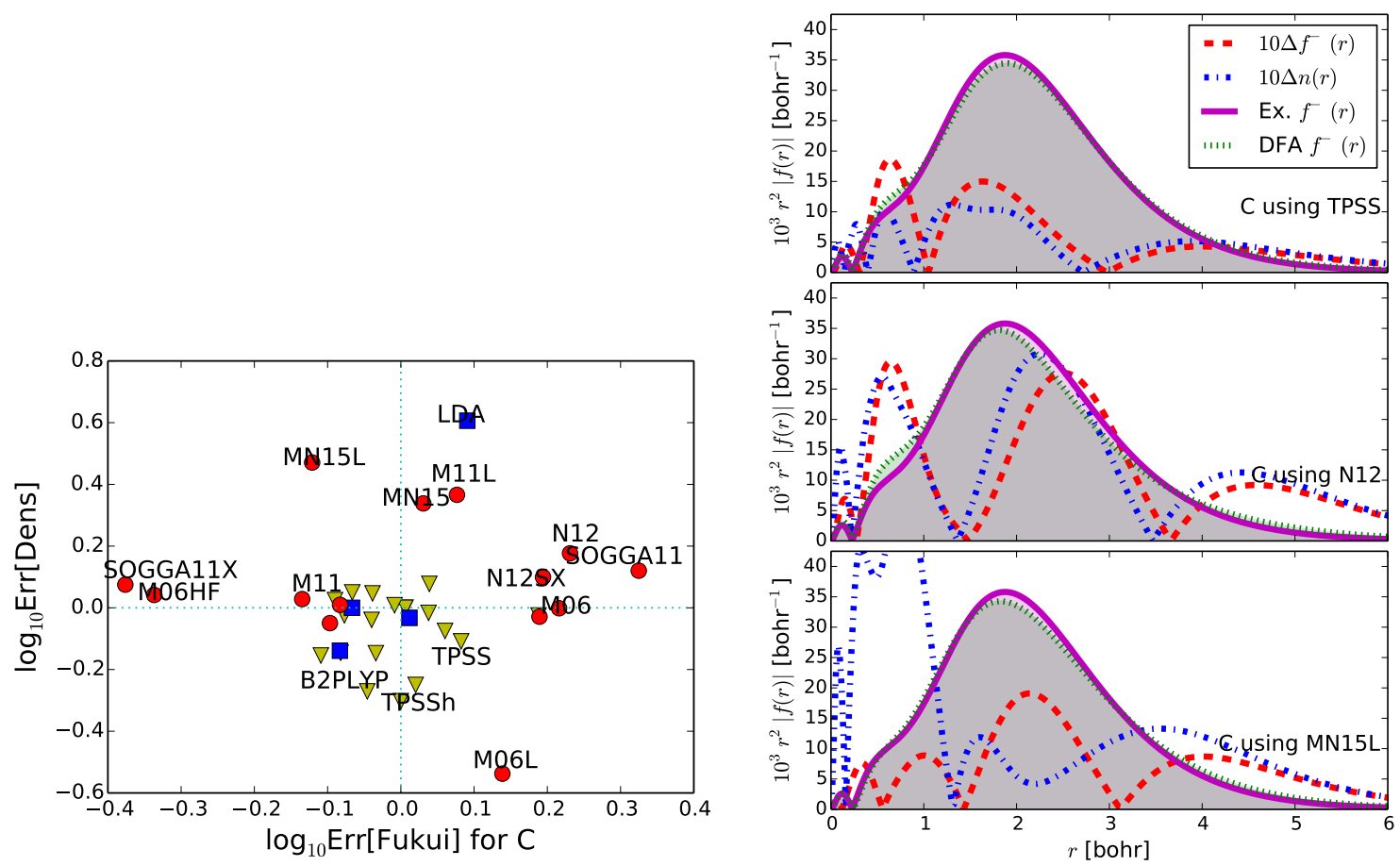

Figure 2: Error in the Fukui function versus errors in the density for $\mathrm{C}$ (top). Dotted lines indicate the median on each axis. Minnesota functionals are shown as circles, LDA, PBE, B3LYP and HSE06 are squares, other functionals are triangles. Only the best and worst DFAs on both metrics are labelled. Also (bottom), errors in the density $n(r)$ compared with errors in the Fukui function $f^{-}(r)$ for exemplar balanced (TPSS - OK $n$ and $f^{-}$), density-skewed (N12 - OK $n$, poor $f^{-}$) and Fukui-skewed (MN15L - OK $f^{-}$, poor $n$ ) DFAs.

respectively

$$
\begin{gathered}
\operatorname{Err}[\text { Dens }]^{2} \propto 4 \pi \int\left[n_{\mathrm{DFA}}(r)-n(r)\right]^{2} r^{2} d r, \\
\operatorname{Err}[\text { Fukui }]^{2} \propto 4 \pi \int\left[f_{\mathrm{DFA}}^{-}(r)-f^{-}(r)\right]^{2} r^{2} d r .
\end{gathered}
$$

Also used are errors in the energy $\operatorname{Err}[\operatorname{En}] \propto\left|E_{\text {DFA }}^{\text {atom }}-E^{\text {atom }}\right|$ and ionization potential $\operatorname{Err}[\mathrm{IP}] \propto\left|E_{\mathrm{DFA}}^{\text {atom }}-E_{\mathrm{DFA}}^{\text {cation }^{+}}-E^{\text {atom }}+E^{\text {cation }^{+}}\right|$. For comparison purposes, values are scaled by their median (on all tested DFAs) for each species and metric, so that they are appropriately normalized.

Figure 2 (top) shows one of the most important results from this study: approximations 
that are good at densities are not necessarily good at getting density differences (Fukui functions) and vice versa. Indeed the lower left quadrant (good Fukui functions and densities) is almost empty, except near the median. Simultaneous satisfaction of both densities and their differences is clearly difficult.

This means that poor reproduction of atomic densities (in which errors in core contributions will typically dominate over errors in frontier contributions) does not necessarily lead to poor reproduction of the Fukui function (which is instead dominated by frontier contributions), even though both are density-like quantities that one might expect to be similarly treated by an approximation. There is an analogy to this situation, however - making a fitting approximation to a function. The ideal is to have a fit that works everywhere. But in practice one often employs a fit that is valid in a given regime, such as for small or large values of the input parameter. It is possible that the lack of apparent correlations between (core) densities and Fukui functions points to a similar regime selection in the DFAs, with some doing better in the core regime, and others in the frontier regime.

Integrated metrics can hide details, however. To provided a more detailed perspective on behaviours, Figure 2 (bottom) shows the Fukui function under three selected exemplar approximations, and the respective errors in the Fukui function and density for the same DFAs. The DFAs include a case (TPSS) that gave a decent Fukui function and good density, a density-skewed case (N12) that gave a decent density but poor Fukui function, and a Fukuiskewed case (MN15L) that gave a good Fukui function but poor density. Note that MN15L correctly captures the near-core $\left(r \approx 1\right.$ bohr) behaviour in $f^{-}$much better than the other two DFAs, leading to its strong success on that measure. This brings home the delicate balancing act in densities - MN15L is the worst performer of the three for densities in this same region.

To provide insight into how different metrics relate to one another, Figure 3 shows errors in the ionization potential versus errors in the Fukui function. One might expect that improving density differences (Fukui functions) might also lead to improved energy differences 

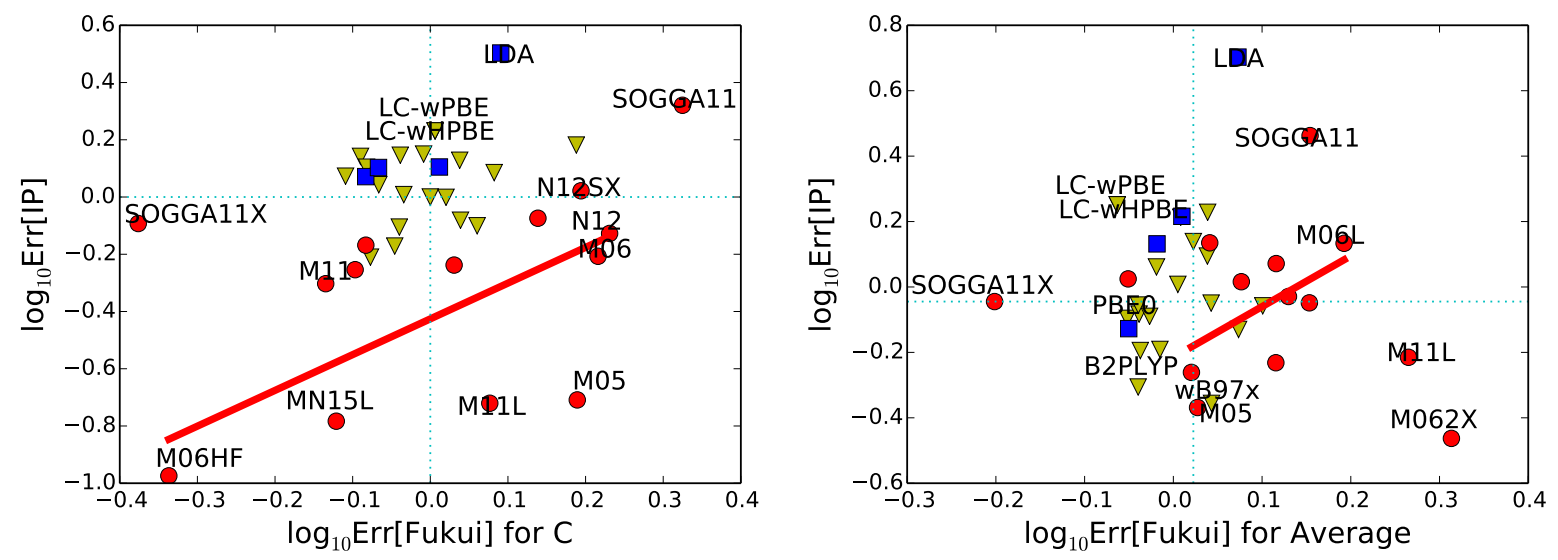

Figure 3: Errors in ionization potentials versus errors in Fukui functions for C (left), and averaged (right) across Li, C and F. Dotted lines indicate the median on each axis. Red lines indicate fits on selected Minnesota DFAs.

(IPs). Such a result will show in this plot as a straight line behaviour.

Indeed the empirically optimized Minnesota functionals M05, M052X, M06, M06L, M06HF, N12, N12SX, MN15 and MN15L (not all labeled - we exclude methods that were difficult to converge from this list, and leave off the outlier cases M062X and SOGGA11X) do almost form a line in the average plot, and follow the same trend in the $\mathrm{C}$ plot, albeit with more noise. This suggests that, for that family of approximations at least, the IP and Fukui function are related, and perhaps hints at strategies to improve future approximations.

By contrast, there seems to be little correlation between Fukui errors and IP errors overall. This may indicate that strict limiting criteria improve densities and energies together, as suggested in previous analysis. ${ }^{6}$ But that they are less good at improving differences. This conclusion also fits with previous results ${ }^{11}$ on atoms showing that $\mathrm{PBE}^{9}$ (as an exemplar functional designed around various density limits) captures closed-shell atomic physics much better than open-shell physics.

Finally, the "star performers" in the analysis are clearly the optimized SOGGA11X, ${ }^{22}$ the hybrid-meta-GGA TPSSh, ${ }^{23}$ and double-hybrid B2PLYP ${ }^{24}$ (although readers are advised to read the cautionary note about B2PLYP in the Supporting Information before drawing conclusions), all of which are constructed according to various exact constraints. Their 
Table 1: Ranks of star performer DFAs.

\begin{tabular}{|l|cc|cc|}
\hline DFA & \multicolumn{2}{|c|}{ Dens. rank. } & \multicolumn{2}{|c|}{ Fuk. rank. } \\
& C & Avg. & C & Avg. \\
\hline B2PLYP & 3 & 1 & 14 & 6 \\
\hline TPSSh & 2 & 2 & 19 & 11 \\
\hline SOGGA11X & 29 & 27 & 1 & 1 \\
\hline
\end{tabular}

rankings on different species and metrics are shown in Table 1, highlighting their broad success in getting densities and/or Fukui functions in chemically-relevant cases. TPSSh was also a high achiever in prior work, ${ }^{6}$ indicating its high overall quality. But SOGGA11X was on neither the naughty nor nice list, previously, and its non-hybrid counterpart SOGGA11 is one of the worst performers here for both densities and Fukui functions. It is worth noting that SOGGA11X achieves its success through an outstanding performance on the Fukui function for Li and C. Taking its maximum normalised error (from F) relegates it to a mid-range performer, more in line with previous results.

To summarise, this manuscript reports an analytic approach which aims to provide a chemically-relevant test of DFAs' ability to reproduce electron densities. Rather than testing chemically-irrelevant closed-shell atoms and ions this work focuses on Li, C (especially) and F, atoms which are involved in or exemplify many reactions. Rather than straight densities and their derivatives, tests are reported for left Fukui functions, a conceptual model for bonding physics based on density differences. Thus, the analysis is likely to identify successes and problems for actual chemical systems.

Correlations between the quality of densities and Fukui functions are shown to be weak, including in the all important $\mathrm{C}$ atom. In some cases methods give excellent densities but mediocre Fukui functions. In other cases the opposite occurs. This is consistent with the result recently reported by Brorsen et $a l^{25}$ that "[some of the] weakest performing functionals for predicting atomic densities, [...] perform significantly better than expected for the computation of diatomic densities". Neither correlates strongly with energies overall. Three star performers are found - TPSSh, SOGGA11X and B2PLYP. These approximations give 
exceptional densities and/or Fukui functions, suggesting that they correctly capture densities in the core and frontier regimes.

Many of the highly-parameterised Minnesota functionals are shown here to perform well, in contrast to previous analysis. ${ }^{6}$ This likely reflects a tendency of parametrisation to improve frontier density differences, sometimes at the expense of core densities. This is exemplified by the functional MN15L on $\mathrm{C}$, which has only moderate success at reproducing the density but captures the Fukui function very well. Correlations between quality of energy and density differences also suggest that optimization strategies might be improved by considering chemically relevant density differences. This might be a good compromise between unconstrained fitting, and flexibility.

This work thus vindicates, to some degree, the strategy of semi-empirical optimization. Although the star performance of SOGGA11X also highlights the importance of exact constraints. The author agrees with the argument that good functionals should aim for good energies and densities. But is a method that sacrifices unimportant core densities but captures chemically important density differences "straying from the path towards the exact functional" "6? Or just taking an efficient shortcut? This work supports the latter perspective.

\section{Acknowledgement}

The author thanks Dario Rocca for stimulating discussion, Yun Wang for suggesting $\mathrm{CF}_{4}$ as an example, and Julien Toulouse for suggesting double-hybrids. T.G. received exemplary computing support from the Griffith University Gowonda HPC Cluster.

\section{Supporting Information Available}

Additional data, including a full list of DFAs, for all tested functionals is available in the supporting information. Some additional discussion on theory is also included. This material is available free of charge via the Internet at http://pubs.acs.org/. 


\section{References}

(1) Hohenberg, P.; Kohn, W. Inhomogeneous Electron Gas. Phys. Rev. 1964, 136, B864B871.

(2) Kohn, W.; Sham, L. J. Self-Consistent Equations Including Exchange and Correlation Effects. Phys. Rev. 1965, 140, A1133-A1138.

(3) Perdew, J. P.; Schmidt, K. In Density Functional Theory and Its Application to Materials; Doren, V. V., Alsenoy, C. V., Geerlings, P., Eds.; AIP: Melville, New York, 2001.

(4) Heyd, J.; Scuseria, G. E.; ; Ernzerhof, M. Erratum: Hybrid functionals based on a screened Coulomb potential. J. Chem. Phys. 2006, 124, 219906.

(5) Verma, P.; Truhlar, D. G. HLE16: A Local Kohn-Sham Gradient Approximation with Good Performance for Semiconductor Band Gaps and Molecular Excitation Energies. J. Phys. Chem. Lett. 2017,

(6) Medvedev, M. G.; Bushmarinov, I. S.; Sun, J.; Perdew, J. P.; Lyssenko, K. A. Density functional theory is straying from the path toward the exact functional. Science $\mathbf{2 0 1 7}$, $355,49-52$.

(7) Kepp, K. P. Comment on "Density functional theory is straying from the path toward the exact functional". Science 2017, 356, 496-496.

(8) Medvedev, M. G.; Bushmarinov, I. S.; Sun, J.; Perdew, J. P.; Lyssenko, K. A. Response to Comment on "Density functional theory is straying from the path toward the exact functional". Science 2017, 356, 496-496.

(9) Perdew, J. P.; Burke, K.; ; Ernzerhof, M. Generalized gradient approximation made simple. Phys. Rev. Lett. 1996, 77, 3865-68. 
(10) Tao, J. M.; Perdew, J. P.; Staroverov, V. N.; ; Scuseria, G. E. Climbing the density functional ladder: Nonempirical meta-generalized gradient approximation designed for molecules and solids. Phys. Rev. Lett. 2003, 91, 146401.

(11) Burke, K.; Cancio, A.; Gould, T.; Pittalis, S. Locality of correlation in density functional theory. J. Chem. Phys. 2016, 145, 054112.

(12) Gould, T.; Toulouse, J. Kohn-Sham potentials in exact density-functional theory at noninteger electron numbers. Phys. Rev. A 2014, 90, 050502.

(13) Burke, K. Perspective on density functional theory. J. Chem. Phys. 2012, 136, 150901.

(14) Becke, A. D. Perspective: Fifty years of density-functional theory in chemical physics. J. Chem. Phys. 2014, 140, 18A301.

(15) Jones, R. O. Density functional theory: Its origins, rise to prominence, and future. Rev. Modern Phys. 2015, 87, 897.

(16) Yang, W.; Parr, R. G.; Pucci, R. Electron density, Kohn-Sham frontier orbitals, and Fukui functions. J. Chem. Phys. 1984, 81, 2862-2863.

(17) Seidl, A.; Görling, A.; Vogl, P.; Majewski, J. A.; Levy, M. Generalized Kohn-Sham schemes and the band-gap problem. Phys. Rev. B 1996, 53, 3764-3774.

(18) Parr, R. G.; Yang, W. Density functional approach to the frontier-electron theory of chemical reactivity. J. Am. Chem. Soc. 1984, 106, 4049-4050.

(19) Hirshfeld, F. L. Bonded-atom fragments for describing molecular charge densities. Theoretica Chimica Acta 1977, 44, 129-138.

(20) Becke, A. D. A new mixing of Hartree-Fock and local density-functional theories. J. Chem. Phys. 1993, 98, 1372-77. 
(21) Zhao, Y.; Truhlar, D. G. The M06 suite of density functionals for main group thermochemistry, thermochemical kinetics, noncovalent interactions, excited states, and transition elements: two new functionals and systematic testing of four M06-class functionals and 12 other functionals. Theor. Chem. Accounts 2008, 120, 215-241.

(22) Peverati, R.; Truhlar, D. G. A global hybrid generalized gradient approximation to the exchange-correlation functional that satisfies the second-order density-gradient constraint and has broad applicability in chemistry. J. Chem. Phys. 2011, 135, 19110.

(23) Staroverov, V. N.; Scuseria, G. E.; Tao, J.; Perdew, J. P. Comparative assessment of a new nonempirical density functional: Molecules and hydrogen-bonded complexes. $J$. Chem. Phys. 2003, 119, 12129.

(24) Grimme, S. Semiempirical hybrid density functional with perturbative second-order correlation. J. Chem. Phys. 2006, 124, 03410.

(25) Brorsen, K. R.; Yang, Y.; Pak, M. V.; Hammes-Schiffer, S. Is the Accuracy of Density Functional Theory for Atomization Energies and Densities in Bonding Regions Correlated? J. Phys. Chem. Lett. 2017, 


\section{Graphical TOC Entry}

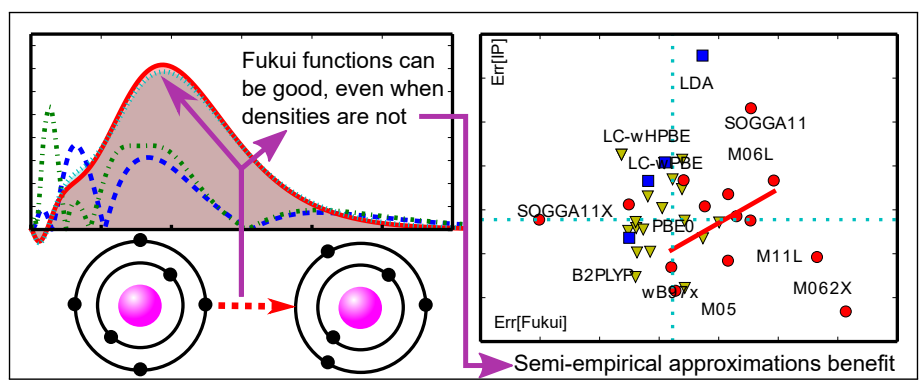

